Cinémas

Revue d'études cinématographiques

Journal of Film Studies

\title{
Enjeux interprétatifs de la féminité dans l'écriture de Souleymane Cissé
}

\section{Samuel Lelièvre}

Volume 11, numéro 1, automne 2000

Écritures dans les cinémas d’Afrique noire

URI : https://id.erudit.org/iderudit/024834ar

DOI : https://doi.org/10.7202/024834ar

Aller au sommaire du numéro

Éditeur(s)

Cinémas

ISSN

1181-6945 (imprimé)

1705-6500 (numérique)

Découvrir la revue

Citer cet article

Lelièvre, S. (2000). Enjeux interprétatifs de la féminité dans l'écriture de Souleymane Cissé. Cinémas, 11(1), 61-76. https://doi.org/10.7202/024834ar

\section{Résumé de l'article}

Dans tout le cinéma de Cissé se retrouvent deux thèmes : l'Afrique et la femme. Si bien qu'on pourrait considérer que s'y développent des thèses panafricaines ou féministes. Mais à eux seuls ces cadres idéologiques ne permettent pas de saisir la relation qu'entretiennent, selon le Malien, l'Afrique et la femme avec la féminité. Dans un premier temps, cette relation sera abordée à travers la description concrète que fait le cinéaste de la condition des femmes africaines; dans un second temps, elle sera identifiée au passage à un discours plus abstrait du cinéaste sur la condition féminine de l'Afrique. On comprendra ce passage dans la mesure où on aura d'abord considéré l'écriture cinématographique de Cissé sur la féminité comme porteuse d'un sens métaphorique. 


\title{
Enjeux interprétatifs de la féminité dans l'écriture de Souleymane Cissé
}

\section{Samuel Lelièvre}

\begin{abstract}
RÉSUMÉ
Dans tout le cinéma de Cissé se retrouvent deux thèmes: l'Afrique et la femme. Si bien qu'on pourrait considérer que s'y développent des thèses panafricaines ou féministes. Mais à eux seuls ces cadres idéologiques ne permettent pas de saisir la relation qu'entretiennent, selon le Malien, l'Afrique et la femme avec la féminité. Dans un premier temps, cette relation sera abordée à travers la description concrète que fait le cinéaste de la condition des femmes africaines; dans un second temps, elle sera identifiée au passage à un discours plus abstrait du cinéaste sur la condition féminine de l'Afrique. On comprendra ce passage dans la mesure où on aura d'abord considéré l'écriture cinématographique de Cissé sur la féminité comme porreuse d'un sens métaphorique.
\end{abstract}

\section{ABSTRACT}

The recurrence of two themes (Africa and women) in Cissé's cinema as a whole would tend to suggest that his body of work develops pan-African as well as feminist theses. Yet these ideological frameworks alone do not suffice to understand the relationship which, according to the Malian director, Africa and women have with femininity. This relationship will first be examined through the concrete description by the filmmaker of the condition of African women; it will then be identified with Cissé's movement towards a more abstract discourse on the condition of women in Africa. 
This movement can be understood insofar as the director's filmic écriture on femininity has first been considered as the bearer of metaphoric meaning.

\section{Enjeux interprétatifs de la féminité}

\section{dans l'écriture de Souleymane Cissé}

Souleymane Cissé est couramment considéré comme un cinéaste s'attachant à décrire la situation de la femme africaine et l'importance de son rôle dans ce contexte culturel particulier ${ }^{1}$. Pour étudier son cinéma, les informations apportées par les sciences sociales pourraient a priori se révéler utiles. Le but de cet article n'est pourtant pas de déployer une vaste érudition en ayant la prétention de présenter l'ensemble des significations (anthropologiques, sociologiques, psychologiques, etc.) auxquelles la représentation de la femme dans la filmographie de Cissé peut donner lieu. Il convient surtout de rappeler ici que ce cinéaste, comme la plupart de ses collègues africains, n'a jamais fait des films qui ne s'adresseraient qu'à des spécialistes de l'Afrique. Il souhaite au contraire offrir une image du Mali et de l'Afrique par l'intermédiaire d'un regard qui se veut le moins distant et le plus respectueux possible des cultures et des peuples.

Une telle éthique n'est évidemment pas incompatible avec les approches scientifiques; n'en déplaise aux tenants d'un discours nationaliste, les recherches à la fois "occidentales" et "africaines" qui se font actuellement en Afrique se situent le plus souvent dans cette perspective. Simplement, la pratique du cinéaste malien est, du début à la fin, issue d'une relation d'altérité, non seulement entre l'Afrique et l'Occident, mais aussi entre le cinéaste et son continent. En fait, Cissé se trouve le plus souvent dans une position paradoxale. Il veut d'un côté rendre compte de réalités vécues parfois de manière douloureuse, réalités impossibles à décrire de manière objective. D'un autre côté, pour que son cinéma trouve une certaine légitimité, il doit chercher à être vu et compris de ceux qui sont étrangers aux réalités en question; il travaille en effet dans un contexte où l'activité artistique est considérée tantôt comme un luxe, tantôt comme incompatible avec la tradition orale et l'esprit communautaire des sociétés africaines. 
Pour mieux comprendre l'écriture cinématographique de Cissé, j'aimerais la rapporter d'une part aux réalités qu'elle met en activité et d'autre part à la réception qui peut en être faite. Si j'utilise l'expression "mise en activité", c'est d'une part pour insister sur l'idée d'une culture en train de se faire et d'autre part pour rapporter cette action au "réalisme" de l'écriture cinématographique de Cissé. Je décrirai ainsi le sens global qui peut être attaché au thème de la féminité dans son cinéma et je montrerai que la perception de ce sens global est indissociable d'une lecture interprétative, autrement dit d'une approche ouverte n'exigeant aucune compétence particulière.

Cette lecture ne se fait pas pour autant dans le vide. Ainsi, le discours de Cissé sur l'Afrique et sur la femme doit être rapporté à deux cadres idéologiques et théoriques: le panafricanisme, presque incontournable pour comprendre les cinémas africains, et plus secondairement le féminisme. Je ne considère pas que ces cadres s'opposent ou se complètent, mais qu'ils sont à la fois liés et distincts, comme sont liés et distincts, de Den Muso (1975) à Waati (1995), les regards portés par le cinéaste sur la femme africaine. Je souhaite ainsi retracer la continuité historique à travers laquelle Cissé met en film l'Afrique et la femme.

Dans un premier temps, la mise en activité d'une féminité africaine peut être abordée à travers la description que fait Cissé de la vie quotidienne des femmes au Mali, dans une perspective qui se veut réaliste, voire même didactique. Elle peut ensuite m'amener aux abords d'un discours panafricain porteur d'une conception avant tout matriarcale de la société. Ce cinéma ferait alors référence de manière implicite à la conception de Cheick Anta Diop selon laquelle la société matriarcale est un dénominateur commun à tout le continent africain (1959, p. 163-169). C'est dans le passage d'un regard relativement réaliste sur la condition de la femme africaine à un discours plus abstrait où le cinéaste traite de la condition féminine de l'Afrique que la représentation de la féminité revêt un sens métaphorique.

Dans l'analyse qui va suivre, je ne considère pas ce sens métaphorique comme un simple transfert sémantique entre la femme africaine et l'Afrique. Il convient d'aller au-delà de ce formalisme, non seulement pour apprécier en dehors d'un cadre 
strictement idéologique le regard de Cissé sur la femme et sur l'Afrique, mais aussi pour voir en quoi ce thème oblige les études cinématographiques à se déterminer par rapport à une théorie de l'interprétation. J'ajouterai donc que mon analyse ne peut appartenir ni à une idéologie panafricaine (ou "afrocentriste") ni à une idéologie féministe. Le panafricanisme, avec l'importance qu'il redonne à la femme, peut jeter un voile sur la singularité du cinéma de Cissé, il peut en bloquer toute lecture non africaine. D'un autre côté, une lecture féministe serait bien en peine de trouver chez Cissé un discours théorique et systématique sur l'exploitation des femmes ou sur la féminité.

En réalité, il apparaît progressivement que la distinction entre une image concrète de la femme et un discours plus abstrait sur l'Afrique est beaucoup moins facile à faire: le transport de l'un à l'autre de ces niveaux sémantiques s'effectue aussi à travers le passage d'une image de la société patriarcale où des éléments matriarcaux perdurent, à la mise en activité filmique d'une société où la femme aurait un rôle éminent à jouer. Ce sens métaphorique plus complexe m'amènera à proposer finalement un rapprochement avec l'herméneutique élaborée par Paul Ricœur. Il s'agit de rendre malgré tout possible la compréhension des relations d'altérité - celle de Cissé avec sa communauté culturelle, celle de mon interprétation avec le cinéma de Cissé dans un réseau complexe de significations. Autrement dit, il s'agit de comprendre le sens global d'une complexité qui, dans un même mouvement, produit mes interprétations du cinéma de Cissé et est produit par ces diverses interprétations.

\section{La condition de la femme africaine}

Den Muso, le premier long métrage de Cissé, réunit à sa façon le rapport au réel qui détermine un sens politique et le sens plus métaphysique qui est attaché à la féminité dans un contexte africain. Cette œuvre décrit le parcours tragique d'une jeune Malienne muette qui, lorsqu'elle tombe enceinte, est rejetée à la fois par sa famille et par le père de son enfant; elle met finalement le feu à la hutte de son ancien petit ami avant de se donner la mort. Le cinéaste (Cissé, 1978) explique son intention en ces termes: 
J'avais voulu exposer dans ce film le cas de très nombreuses "filles-mères" que l'on rencontre aujourd'hui dans la pllupart des pays africains, traînant dans les rues, rejetées de partout, constituant une sorte de nouvelle caste d'intouchables à cause de principes sociaux surannés; les familles demeurent attachées à d'anciennes convictions rnorales sans vouloir aborder lucidement le problème de leurs enfants... J'ai voulu mon hérö̈ne muette pour symboliser, en plus très sommairement, une évidence: que chez nous les femmes n'ont pas le droit à la parole. J'estime que certaines traditions, qui ont pu avoir une importance historique, doivent aujourd'hui être abandonnées: notamment tous les préjugés attachés à l'origine familiale, aux rapports dans le mariage, etc.

En dehors du panafricanisme, l'intérêt de Souleymane Cissé pour la condition de la femme africaine ne correspond au point de vue d'aucune idéologie dominante. Le cinéaste ne réduit pas la question de la féminité à un essentialisme machiste - la femme comme être humain proche de la nature - ni à un antihumanisme tiers-mondiste - la condition pénible des femmes africaines comme résultat du capitalisme et de l'impérialisme économique. Dans ce cinéma, le thème de la féminité donne lieu à un développement tel que je ne peux le limiter au cadre relativement abstrait du panafricanisme: le cinéaste ne se contente pas de faire une référence insistante à des femmes qui seraient par ailleurs éloignées de lui; il les place véritablement au centre de ses films.

Dès ses débuts, il déjoue ainsi les critiques qui, d'un point de vue politique ou métaphysique, occidental ou africain, échouent à rendre compte du rôle de la femme dans la société malienne et africaine. Il est en particulier remarquable que ni la critique tiers-mondiste ni la critique anti-tiers-mondiste ne remettent en cause la conception de Souleymane Cissé : la première, parce qu'elle pense faire table rase d'une tradition qui accorde à la femme un rôle éminent; la seconde, parce qu elle réduit généralement les tiers-mondismes, et le point de vue politique qu'ils induisent, à un comportement méprisant envers la femme (Bruckner, 1983, p. 253-254). Si je devais suivre ces discours, je serais obligé de définir le rapport à la féminité dans le cinéma de 
Cissé comme à la fois tiers-mondiste - parce qu'il procède aussi d'une critique de la tradition - et comme anti-tiersmondiste - parce qu'il revêt aussi un sens métaphysique. En somme, je serais tenu de définir cette relation comme une contradiction. Cette façon de procéder n'est pas interdite, mais elle a le gros inconvénient de simplifier, voire de négliger, le sens à la fois politique et métaphysique du cinéma de Souleymane Cissé, notamment à travers ce thème de la féminité.

Le cinéaste malien est musulman. Ce qui ne l'empêche pas de s'inspirer du marxisme - c'était notable à ses débuts - et d'être à l'affût de possibles avancées sociales. Comme beaucoup de cinéastes africains, ou même d'intellectuels africains, Cissé n’a jamais fait de P'islam un absolu culturel. Suivant la thèse d'Ali Mazrui, il convient d'admettre que cette position n'est pas contradictoire ou incohérente; elle est le reflet de la complexité qui caractérise les contextes culturels africains et leur évolution ${ }^{2}$. Le thème de la féminité est impensable hors de cette complexité irréductible à la critique tiers-mondiste comme anti-tiers-mondiste. Alors que l'histoire a révélé les limites de la première - qui continue néanmoins d'avoir un lien historique avec les cinémas africains -, la dernière adopte un point de vue externe au contexte africain, ce qu'elle appelle l'« européocentrisme» (Bruckner, 1983, p. 282286), y compris quand elle parle d'une proximité avec le tiersmonde. Depuis que ce discours est devenu le prétexte à une critique idéologique de l'Islam, il n'est d'aucun soutien pour penser la complexité du contexte africain. Or, je le répète, chez le cinéaste malien, le thème de la féminité n'est pensable qu'à l'intérieur de cette complexité; la condition de la femme peut ainsi refléter quelques-uns des problèmes de la société malienne.

Décrivant différents domaines de la vie en société, les films de Cissé montrent en effet que les femmes ont parfois des comportements assez inattendus. Le premier domaine concerné, c'est celui du travail. Dans Baara (1978), M'Batoma n'exerce aucune profession parce que son ingénieur de mari ne le veut pas "par là, j’ai voulu indiquer que [l'ingénieur] n'était pas un héros positif", explique le cinéaste (Ruelle et Tournes, 1978, p. 30) tandis que Djénéba, qui n'a pas fait d'études, dirige d'une main de fer un commerce florissant. Le second domaine, c'est celui 
du mariage. Djénéba semble avoir épousé un riche industriel malien afin d'asseoir son activité de commerçante; d'un autre côté, elle réserve son cœur à ses amants. D'un point de vue féministe, Farida Ayari la considère "comme l'un des personnages féminins les plus engagés du cinéma africain " (p. 183). Elle représente surtout une catégorie de femmes qui, ayant d'une certaine façon compris le mode de fonctionnement de la société dans laquelle elle vit, ne veut pas en supporter tous les inconvénients. Dans Finyé (1982), il est plutôt question de la polygamie et des problèmes qu'elle pose indubitablement aux sociétés africaines contemporaines: le film expose clairement cette question à travers des scènes de disputes entre les femmes de Sangaré ou entre le gouverneur et l'une de ses femmes. Je pourrais donc émettre l'hypothèse que cette conception du mariage n'est plus conforme aux désirs des femmes. Ce qui conduit finalement au domaine de la sexualité ou plus précisément, de Cinq Jours d'une vie (1972) à Finyé, à celui de la morale sexuelle.

Néanmoins, ce dernier domaine renvoie toujours au rôle social des femmes. Il faut sortir d'une description de ce rôle pour percevoir dans les films de Cissé une dimension psychologique qui est généralement absente des cinémas africains. Cette dimension résulte d'une concentration du regard cinématographique sur les différents aspects de la vie sociale des femmes au quotidien. Dans Baara, l'usage du gros plan peut ainsi être lu comme décrivant les différentes étapes émotionnelles vécues par M'Batoma (la fernme de l'ingénieur). Au niveau le plus dramatique de l'écriture cinématographique de Cissé, cet usage aboutit par exemple aux plans révélant son inquiétude sur le trajet de l'usine, pendant que son mari se fait assassiner.

\section{La condition féminine de l'Afrique}

À sa manière, le thème de la féminité révèle qu'il est difficile d'approcher un cinéma africain sans commettre de contresens et sans risquer, à travers l'indifférence d'un regard étranger ou autochtone, de manquer certains problèmes qu'il soulève. La complexité et l'importance de ce thème dans le cinéma de Souleymane Cissé tiennent au fait qu'il constitue un trait d'union entre un patriarcat contemporain et le matriarcat des traditions 
africaines, entre l'islam (ou ailleurs le christianisme) et le panafricanisme. Au-delà d'un questionnement sur un contexte culturel, le thème de la féminité doit ainsi être situé entre un niveau social et un niveau sémantique: il s'agit de dégager un sens global qui entretient nécessairement un rapport avec l'universalité, sans pour autant renoncer à un ancrage culturel et traditionnel spécifique.

Traiter du problème de la polygamie, par exemple, éloigne le récepteur d'une certaine universalité tout en le rapprochant du contexte africain. Sur cette question de la représentation de la polygamie, Manthia Diawara considère qu'il est nécessaire de savoir que ce type de mariage est traditionnellement source de prestige. Celui-ci se mesure au nombre de femmes que possède le mari. Or, dans Finyé, il est plutôt source de conflits et de luttes de pouvoir. Par ailleurs, la plus jeune femme du gouverneur - Agna - boit, fume, et a une liberté qui n'existe pas dans un mariage polygamique traditionnel. Voulant malgré tout répondre à cette double contrainte théorique posée par la relation entre un niveau sémantique et un niveau social, Manthia Diawara (1996, p. 212) arrive à la conclusion suivante quant à la représentation de la polygamie dans Fynié:

Une compréhension de la culture autochtone (signes anthropologiques) est nécessaire pour apprécier le jeu du mari autoritaire et phallocrate, ou celui des femmes, de la plus jeune à la plus âgée. On doit aller au-delà de la conception simpliste d'un art (le cinéma) qui, en Afrique, aurait seulement un rôle fonctionnel, et voir, par exemple dans la scène comique de la dispute entre les femmes du gouverneur, comment l'esthétique est surdéterminée par la question de la polygamie.

Bien qu'étant surdéterminé par la culture, le rapport de l'esthétique avec le sens global du cinéma de Cissé (et de notre interprétation de ce cinéma) impose que cette compréhension des "signes anthropologiques" n'appartienne ni seulement à l'esthétique ni seulement à la culture, mais à la fois à l'esthétique et à la culture. L'ambition du cinéaste n’a jamais été de n'être intelligible que de ses compatriotes. Une des stratégies du cinéaste malien pour amener à une compréhension de ces signes anthro- 
pologiques est de faire du continent africain en général un élément de plus en plus central dans son œuvre. Dans Waati (1995), Cissé se sert de la féminité comme d'une métaphore de ce thème: il s'agit de considérer ce que je pourrais appeler la condition féminine de l'Afrique.

Cette expression de "condition féminine de l'Afrique" renvoie à un sens propre et à un sens figuré. Elle peut être rapportée au sens propre d'une mise en activité imagée réaliste de la féminité en Afrique; une justification par les faits qui rapprocherait d'une certaine vérité. Dans cette perspective, il s'agit de considérer la condition de la femme africaine comme représentative de celle de l'Afrique. L'expression renvoie également au sens figuré et panafricain de la féminité considérée comme le dénominateur commun à toute l'Afrique. Dans cette perspective, il s'agit de faire une analogie entre des femmes qui «sont encore et toujours victimes, sous la domination de l'homme, alors même qu'elles restent la base de la société, de la famille" (Elhelm et Waldmann, 1988, p. 6), et un continent qui serait encore et toujours victime, sous la domination de l'Occident, alors qu'il constituerait la base de l'humanité.

A mon sens, c'est cette dernière perspective qui fait problème. Elle suppose en effet l'acceptation de thèses panafricaines dont certaines doivent être combattues. Il n'entre pas dans le cadre de cet article d'analyser les problèmes posés par le panafricanisme de Cheick Anta Diop, pour citer le représentant le plus influent de ce courant d'idées. Il convient néanmoins de rappeler qu'il a fait de l'Afrique le «berceau de l'humanité" — ce qu'enseigne la paléontologie, mais qui peut être un jour invalidé —, qu’il est obsédé par la définition d' "une identité nègre» et que, par conséquent, ses écrits présentent une conception figée de cette histoire et de cette identité.

Par contre, un film comme Yeelen (1987) montre que la relation de Cissé avec le panafricanisme n’a jamais été le prétexte à un discours sur ce qui serait spécifiquement africain, mais à un discours sur ce qu'est l'histoire de l'Afrique. En situant le dénouement de ce film dans les célèbres falaises de Bandiagara, le Malien fait explicitement référence à la pensée des Dogons. Il se réapproprie le mythe dogon d'une faute originelle. Par des allu- 
sions à une culture immémoriale, à une période d'esclavage et d'aliénation ainsi qu'à une renaissance d'après le chaos, il construit un récit tragique qui est une métaphore assez explicite de l'histoire africaine.

Ce faisant, il se situe aux antipodes du point de vue «différentialiste" et panafricain de Cheick Anta Diop : ce dernier considère en effet que les Dogons ne connaissent pas ce genre de culpabilité, et il y voit de manière très spécieuse une preuve supplémentaire de la nature génétiquement pacifique de l'homme africain (p. 169-170) ${ }^{3}$, par opposition à l'agressivité d'un Occident inventeur de l'esclavage et de la colonisation. À propos de Yeelen - un film qu'il considère comme son chef-d'œuvre Cissé déclare que son objectif est non seulement de critiquer de manière radicale les pouvoirs coutumiers, mais aussi d'évoquer leurs responsabilités dans la colonisation de l'Afrique (Loupias, 1995, p. 22). Le pacifisme (présumé) des sociétés traditionnelles est ainsi clairement intégré au colonialisme européen.

Dans cette perspective, je peux parler d'un sens métaphorique global du cinéma de Cissé, qui peut être divisé en deux points de vue sur la réalité africaine: d'une part, le point de vue actif de la lutte contre une condition dégradante ou inférieure aux hommes; d'autre part, un point de vue plus statique et englobant qui correspond aux apports du monde de la féminité dans la société patriarcale contemporaine. Le premier point de vue est présent dans tous les films de Cissé; il constitue même un des fils rouges les plus solides dans l'œuvre du cinéaste, reliant notamment Den Muso (1975) à Waati (1995). Depuis le premier long métrage, la position du cinéaste a toutefois évolué. Dans Den Muso, Cissé traite plus particulièrement du contexte malien. A l'époque de Waati, Souleymane Cissé considère que les femmes sud-africaines constituent un modèle dans une lutte "qui n'a pas le même niveau dans tous les pays africains" (Boulay et Colmant; 1995). Le second point de vue est déjà présent dans Den Muso, à travers ce que le cinéaste appelle le "mutisme symbolique" de Ténin. Dans tous les cas, il est conduit à regarder son monde avec un peu de recul.

Le silence de Ténin n'est pas seulement là pour exprimer un état de vulnérabilité, mais aussi pour signifier que la femme africaine vit dans un univers rarement compris par les hommes. Un 
univers qui n'est pas seulement complémentaire de l'univers masculin, mais qui est susceptible de l'enrichir, de le porter vers des dimensions qu'il serait bien incapable de découvrir seul. L'un de ces domaines, c'est celui de l'érotisme, et non plus seulement de la sexualité comme enjeu de pouvoir. Cet érotisme est dans un premier temps révélé par la pudeur du cinéaste malien à montrer des corps nus, au-delà de l'impersonnalité d'une pornographie circulant librement par ailleurs. Dans Finyé, Bah n'ose pas se déshabiller devant Batrou; Cissé ne filme que des fragments d'une nudité. Peut-être que le cinéaste est aussi ému que son jeune acteur masculin.

À moins qu'il ne s'agisse d'une autocensure de la part d'un cinéaste à qui un critique africain déclare: "[...] ça fait deux fois que, dans vos films, il y a une scène de toilette entre homme et femme. Cela est contraire à nos traditions" (Touré, 1987, p. 9). Il existe un analogon de cette scène de Finyé dans Yeelen. Le cinéaste se justifie auprès de son critique en déclarant que le spectateur ne les voit pas se laver ensemble. Cette intimité est simplement évoquée. Dans Yeelen, Nianankoro et sa jeune femme ne se lavent pas ensemble; une forme très discrète d'érotisme est néanmoins présente à travers les images d'eaux ruisselantes sur le corps féminin. Cissé n'a pas de gêne particulière à aller sur ce terrain. Par contre, il reste attentif à la réception qui peut en être faite par un public africain.

\section{Pour une lecture interprétative des cinémas africains}

Jusqu’à présent, j’ai essayé de décrire la complexité des relations métaphoriques qui peuvent être établies entre des représentations de l'Afrique et des représentations de la femme africaine. La question demeure cependant de savoir si les approches traditionnelles du cinéma permettent d'analyser ce passage d'une description de la condition de la femme africaine à un discours sur la condition féminine de l'Afrique. Il n'est pas sûr que le récepteur qui cherche à s'appuyer sur les théories esthétiques ou sémiologiques traditionnelles puisse surmonter les problèmes interprétatifs posés par les films de Cissé.

Par rapport à une lecture que j'ai qualifiée d' « interprétative", et qui essaie de théoriser la conception de l'interprétation ainsi 
engagée, les approches qui tendent à limiter la part du sujet interprétant dans l'approche des cinémas africains, conduisent à des impasses. Et cela vaut tant pour les approches formalistes ou "expressives" - "ce signe exprime ceci, cet autre cela" - que pour celles qui s'attachent à décrire un contenu culturel des cinémas africains - "ce film contient telle signification sur la culture et appartient à telle conception de la culture". L'intelligence active du récepteur peut se rendre compte que c'est nécessairement plus complexe, qu'il a aussi un autre rôle à jouer. Et un rôle plus gratifiant, c'est-à-dire qu'on ne peut limiter ni à une sémiologie ou une psychologie de la perception ni à un historicisme qui ne regarderait les films africains que comme des documents.

Le thème de la féminité, parce qu'il est abordé par Cissé de manière essentiellement détournée et métaphorique, montre quelle relation un cinéma africain peut entretenir avec des réalités africaines. Il s'agit d'une relation concrète et complexe, qui revêt parfois un caractère didactique et idéologique, quand elle touche au panafricanisme voire au féminisme, mais quaucun corpus idéologique - tiers-mondisme ou anti-tiers-mondisme, traitement d'un contenu culturel interne aux films - ne permet de saisir de manière satisfaisante. Du point de vue des études cinématographiques traditionnelles, les approches qui n'envisagent les cinémas africains que de l'extérieur, c'est-à-dire qui mettent en œuvre une logique des signes cinématographiques, ne rendent pas davantage compte de cette relation complexe et significative avec les réalités africaines. Il est impossible de trouver dans ces approches "de l'intérieur et de l'extérieur" une réflexion qui toucherait à la fois à la représentation et à la mise en activité de la femme dans les cinémas africains, et aux problèmes d'interprétation posés par ces deux champs théoriques.

L'herméneutique proposée par Paul Ricœur pourrait par contre aider à construire une telle réflexion. Dans un premier temps, je pourrais ainsi considérer que le sens métaphorique, auquel j'ai pu rapporter le thème de la féminité, renvoie d'abord à une vérité métaphorique. Ce qui est métaphoriquement renvoie non seulement à ce qui est comme, mais aussi à ce qui n'est pas, c'est-à-dire à ce qui n'est pas vrai ou qui relève d'une idéologie - par exemple le féminisme ou le panafricanisme - à laquelle 
le spectateur n'est pas obligé d'adhérer (Ricour, 1975). Ce qui est important ici, ce n'est pas le fait d'attribuer aux cinémas africains - et en général à toute œuvre d'art — la faculté de dire une certaine vérité à travers des mensonges ou, plus négativement, d'être l'instrument d'une idéologie : ce sont là des éléments que tout spectateur un peu expérimenté a l'habitude d'intégrer. Certes, le thème de la féminité m'a amené à percevoir la relation métaphorique qui existe entre un point de vue féministe et un point de vue panafricain. Mais j'ai surtout fait face à la complexité d'un sens métaphorique qu'aucune théorie sur la culture ou sur le cinéma ne permet d'épuiser.

Dans un deuxième temps, il convient de voir avec Dudley Andrew que l'herméneutique de Paul Ricœur, par opposition au structuralisme, met en évidence la priorité du discours sur la signification, et de l'interprétation sur la structure (Andrew, 1984, p. 182). Il pourrait alors être pertinent d'appréhender la relation à une identité féminine et africaine selon les termes de cette herméneutique, notamment par rapport à l'élaboration récente de Soi-même comme un autre (Ricœur, 1990). Dans cette dernière perspective, l'identité d'un individu ou d'un peuple ne peut être décrite que du point de vue d'un autre individu ou d'un autre peuple. Non seulement l'activité de l'interprète trouve ainsi une justification éthique, mais le sens métaphorique qu'on a pu rapporter au thème de la féminité procède de l'insertion productive de l'étranger dans le discours. Au-delà d'une identité féminine et africaine, le sens métaphorique de la féminité chez Souleymane Cissé doit aussi m'amener à considérer un devenir féminin et africain. Il s'agit d'une problématique importante à travers laquelle doit être évalué et perçu tout ce que ce cinéma d'Afrique a de créatif et de communicatif, et qui échappe aux discours sur ce qu'il contiendrait ou exprimerait.

À cet égard, mon approche des enjeux interprétatifs entourant le thème de la féminité voudrait introduire une lecture interprétative des cinémas africains en général. Ce qui pourrait paraître dans un premier temps paradoxal n'est en fait qu'un moyen pour les pratiques cinématographiques africaines de véritablement exister et pour les études cinématographiques de mettre leur discours à l'épreuve de ces pratiques. Cette mise en avant du 
travail d'interprétation est le contraire d'un laxisme théorique qui voudrait laisser à n'importe qui la liberté de dire n'importe quoi, et de justifier finalement tous les préjugés et tous les chauvinismes. Les enjeux interprétatifs de la féminité sont de faire entrevoir, non pas un sens global dans le cinéma de Cissé - ce qui ferait de ce cinéma un simple médium pour la transmission d'un message ou d'une idéologie - , mais un sens global $d u$ cinéma de Cissé. A travers une lecture interprétative, le spectateur peut se rendre compte qu'il est nécessaire de considérer en même temps le film, celui qui l'a produit et celui qui l'interprète.

Ce qui relie ces différents acteurs, c'est le principe d'une interprétation selon laquelle l'identité (d'un individu ou d'un peuple) n'est ni mise en avant ni niée, mais renvoie d'abord à une altérité. En m'appuyant sur la théorie de la métaphore de Paul Ricœur, j'ai pu appréhender la relation à une identité en train de se faire. Dudley Andrew (2000) rappelle que cette herméneutique fournit deux autres modes d'approche de cette identité à l'œuvre dans les cinémas africains. Ricœur trace tout d'abord un chemin intermédiaire entre ces extrêmes que sont l'ego cartésien irrévocable d'un côté et le sujet brisé de Nietzsche et de Deleuze de l'autre, et fournit les termes pour comprendre la relation comme une identité à expérimenter. Dudley Andrew fait ensuite remarquer que cette identité peut être appréhendée à travers la relation à ce que Paul Ricœur appelle l'ipse.

En principe, les termes latins idem et ipse peuvent tous deux être traduits en français par "même". Ricœur établit cependant une distinction importante entre ces deux termes: par opposition à l'ensemble de traits immuables d'un récit ou d'une histoire qui ne connaît qu'un changement organique - ce qu'il appelle l'idem -, l'ipse est l'agent de ce récit et de cette histoire, c'est-à-dire d'un devenir. Dans le même temps, l'ipse appartient aux grands achèvements historiques que peuvent être, pour un cinéma malien (et africain), la décolonisation, l'indépendance, et finalement l'émergence de la démocratie. Au sens de Paul Ricœur, l'ipse est ce qui amène de l'altérité dans l'identité.

Dans le cinéma de Cissé, un point de vue panafricain sur l'identité de la femme et de l'Afrique pourrait parfois aller dans le sens d'un idem, autrement dit d'une conception figée de 
l'identité. À travers un engagement politique proche du féminisme et du panafricanisme tout en étant irréductible à ces cadres idéologiques, ce thème de la féminité renvoie surtout à un ipse. De cette façon, une identité à la fois africaine et féminine existe, mais elle ne peut être saisie que par des relations d'altérité. L'écriture cinématographique de Souleymane Cissé reflète le sens à la fois politique et métaphysique qui est à l'œuvre dans ces relations d'altérité. Percevant cette complexité du sens, tout interprète devrait alors chercher à sortir des grilles de lecture préétablies ou fonctionnant en circuit fermé.

\section{Université Marc-Bloch de Strasbourg}

\section{NOTES}

1. Souleymane Cissé vit à Bamako, la capitale du Mali, où il est né en 1940 .

2. Voir à ce sujet l'ouvrage d'Ali Mazrui (1986). Dans l'introduction à son livre sur les cinémas africains, Frank Ukadiké (1994, p. 13) rappelle qu'il est souvent reproché à cet important historien d'être trop proche de l'Islam pour parler en toute objectivité de l'Afrique.

3. Sur le mythe dogon, voir l'ouvrage de Marcel Griaule, Dieu d'eau. Entretiens avec Ogotemmêli (Paris: Fayard, 1966). Tout en considérant que le mérite des travaux d'Anta Diop est de relier la civilisation égyptienne aux civilisations d'Afrique noire, Alain Ricard (1995, p. 20-25) met en garde contre les dangers pouvant découler de son discours sur les races.

\section{OUVRAGES CITÉS}

Andrew, Dudley. "The Roots of The Nomadic: Gilles Deleuze and the Cinema of West Africa", dans Gregory Flaxman (direction), The Brain is the Screen: Deleuze and the Philosophy of Cinema. Minneapolis: University of Minnesota Press, 2000, p. 215-249.

Andrew, Dudley. Concepts in Film Theory. London: Oxford University Press, 1984.

Anta Diop, Cheikh. L'Unité culturelle de l'Afrique. Paris: Éditions Présence africaine, 1959.

Ayari, Farida. «Images of Women", dans Imruh Bakari et Mbye Cham (direction), African Experiences of Cinema. London: British Film Institute, 1996.

Boulay, Anne et Marie Colmant. "On résiste ou on n'existe pas ». Libération, 19 mai 1995.

Bruckner, Pascal. Le Sanglot de l'homme blanc. Tiers-Monde, culpabilité, haine de soi. Paris: Seuil, 1983.

Cissé, Souleymane. "Refléter la trame du quotidien ". Le Monde diplomatique, septembre 1978.

Diawara, Manthia. "Popular Culture and Oral Traditions in African Film", dans Imrah Bakari et Mbye Cham (direction), African Experiences of Cinema. London: British Film Institute (1996), p. 208-214. 
Elhelm, Philippe et Claude Waldmann. "Vers les sources de la lumière". Cinergie (non numéroté) (1988).

Loupias, Bernard. "Pour l'Afrique, le temps presse». Le Nouvel Observateur, 18-24 mai 1995.

Mazrui, Ali. The Africans: A Triple Heritage. Boston: Little, Brown and Company, 1986.

Ricard, Alain. Littératures d'Afrique noire. Des langues aux livres. Paris: CNRS/Karthala, 1995.

Ricour, Paul. Soi-même comme un autre. Paris : Seuil, 1990.

Ricœur, Paul. La Métaphore vive. Paris: Seuil, 1975.

Ruelle, Catherine et Andrée Tournes. "Cissé, Souleymane». Cinémaction, n 3 (novembre 1978).

Touré, Kitia. «Entretien avec Souleymane Cissé». Positif(1987), p. 8-10.

Ukadiké, Frank. Black African Cinema. Berkeley/Los Angeles/London: University of California, 1994. 